\title{
Video Article \\ Simple Generation of a High Yield Culture of Induced Neurons from Human Adult Skin Fibroblasts
}

\author{
Shelby Shrigley ${ }^{1}$, Karolina Pircs $^{1}$, Roger A. Barker ${ }^{1,2}$, Malin Parmar $^{1}$, Janelle Drouin-Ouellet ${ }^{1}$ \\ ${ }^{1}$ Department of Experimental Medical Science, Wallenberg Neuroscience Center, Division of Neurobiology and Lund Stem Cell Center, Lund University

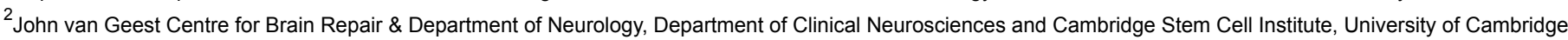

Correspondence to: Janelle Drouin-Ouellet at janelle.drouin-ouellet@med.lu.se

URL: https://www.jove.com/video/56904

DOI: doi:10.3791/56904

Keywords: Neuroscience, Issue 132, Induced neurons, adult human dermal fibroblasts, direct neural reprogramming, human neurons, FACS purification, lentiviral vector

Date Published: 2/5/2018

Citation: Shrigley, S., Pircs, K., Barker, R.A., Parmar, M., Drouin-Ouellet, J. Simple Generation of a High Yield Culture of Induced Neurons from Human Adult Skin Fibroblasts. J. Vis. Exp. (132), e56904, doi:10.3791/56904 (2018).

\section{Abstract}

Induced neurons (iNs), the product of somatic cells directly converted to neurons, are a way to obtain patient-derived neurons from tissue that is easily accessible. Through this route, mature neurons can be obtained in a matter of a few weeks. Here, we describe a straightforward and rapid one-step protocol to obtain iNs from dermal fibroblasts obtained through biopsy samples from adult human donors. We explain each step of the process, including the maintenance of the dermal fibroblasts, the freezing procedure to build a stock of the cell line, seeding of the cells for reprogramming, as well as the culture conditions during the conversion process. In addition, we describe the preparation of glass coverslips for electrophysiological recordings, long-term coating conditions, and fluorescence activated cell sorting (FACS). We also illustrate examples of the results to be expected. The protocol described here is easy to perform and can be applied to human fibroblasts derived from human skin biopsies from patients with various different diagnoses and ages. This protocol generates a sufficient amount of iNs which can be used for a wide array of biomedical applications, including disease modeling, drug screening, and target validation.

\section{Video Link}

The video component of this article can be found at https://www.jove.com/video/56904/

Introduction

Development of efficacious treatments for neurological disorders have been hampered by the limited access to living human brain cells to perform mechanistic studies and functional assays. About a decade ago, this situation radically changed with the development of induced pluripotent stem cell (iPSCs) technology ${ }^{1,2}$. This, combined with a better understanding of the neural differentiation mechanisms occurring during normal human development, has allowed for the generation of defined and diverse neuronal subtypes from patient and disease specific material. With such material, it is now possible to study intracellular mechanisms underlying neurological diseases and the potential of different compounds to alleviate those pathological features ${ }^{3}$.

While iPSCs have been revolutionary to the field of neuroscience, one major drawback of these cells is that their ageing signature is erased during the reprogramming process in such a way that the rejuvenated neuron does not retain the vulnerability associated with aging ${ }^{4,5,6}$. This particular feature of the neurons that are produced may end up being critical for recapitulating many aspects of the intracellular pathogenic cascade, particularly in the case of diseases for which old age is an important risk factor.

Direct neural reprogramming is a technology where a somatic cell is directly converted into an iN without going through a pluripotent intermediate stage. This allows for rapid generation of human neurons in vitro that can be both patient and disease specific. One remarkable characteristic of direct reprogramming is that the starting age of the donor cell is maintained, and with that, its vulnerability to ageing processes such as increased production of oxidative stress ${ }^{4,7}$. As a result, iNs from patients with neurological diseases associated with ageing, such as Alzheimer's and Parkinson's disease, are well suited for a broad range of biomedical applications including disease modeling, drug screening assays, and toxicology studies.

The main caveat that has prevented iNs from patients with neurodegenerative disorders being widely used is that they are not easy to reprogram, and this becomes even more difficult with expansion of the fibroblasts. As a result, generation of iN cells in quantities required for these types of applications has not been achieved until only recently ${ }^{8}$. We have now developed a simple method to reprogram fibroblasts from donors of any age in a very efficient manner. This method combines the forced expression of the neuronal transcription factors Ascl1 and Brn2 with a knockdown of the repressor protein RE1-silencing transcription factor (REST) using a single vector. Here, we describe the different steps leading to the generation of iNs converted from skin fibroblasts biopsied from elderly donors. 


\section{Protocol}

Adult dermal fibroblasts were obtained from the Parkinson's Disease Research and Huntington's disease clinics at the John van Geest Centre for Brain Repair (Cambridge, UK) and used under local ethical approval (REC 09/H0311/88). For details on the skin biopsy sampling procedure, see reference ${ }^{8}$.

\section{Preparation of Skin Fibroblasts for Reprogramming}

1. Using an automated cell thawing system or a $37^{\circ} \mathrm{C}$ water bath, thaw the adult human dermal fibroblasts (aHDFs) and plate 200,000 per T75 flask (count with an automated cell counter) in $10 \mathrm{~mL}$ of fibroblast medium (see Table 1) at $37{ }^{\circ} \mathrm{C}$ in $5 \% \mathrm{CO}_{2}$.

2. Perform a complete medium change with fibroblast medium on the next day.

3. Change the fibroblast medium every $3-4$ days until the cells reach $95 \%$ confluency. NOTE: One confluent flask will contain approximately 1,000,000 cells. The aHDFs can be frozen to build a stock of the cell line (section 2 ) or directly re-plated ready for reprogramming (section 3 ).

\section{Freezing of Skin Fibroblasts}

1. Place a controlled-rate freezing container in a box with ice or in the fridge at $4{ }^{\circ} \mathrm{C}$.

2. Dissociate the cells with $0.05 \%$ trypsin $\left(1.5 \mathrm{~mL}\right.$ per $\mathrm{T} 75$ flask) at $37^{\circ} \mathrm{C}$ for $3-5 \mathrm{~min}$.

3. Add fibroblast medium (containing fetal bovine serum (FBS)) to neutralize the trypsin ( $3 \mathrm{~mL}$ per flush per T75 flask) and collect the detached cells in a $15 \mathrm{~mL}$ tube by flushing out the cells in the flask twice.

4. Count the cells using the automated cell counter; (recommended) freeze approximately 500,000 aHDFs per vial.

5. Spin down the cells at $400 \times \mathrm{g}$ for $5 \mathrm{~min}$.

6. Resuspend the cell pellet in $1 \mathrm{~mL}$ of freezing medium (see Table 1) and transfer into the cryogenic tube. Place the tubes directly into the controlled-rate freezing container.

7. Store the controlled rate freezing apparatus at $-80^{\circ} \mathrm{C}$ overnight. The following day, transfer the tubes to a $-140{ }^{\circ} \mathrm{C}$ freezer and store until needed.

\section{Plating for Reprogramming (Day -1)}

NOTE: It is recommended to use a gelatin coating for short term experiments (up to 30 days); alternatively, for long term experiments it is recommended to start on a poly-L-ornithine, fibronectin and laminin (PFL) coating.

1. $60 \mathrm{~min}$ before plating the aHDFs for reprogramming, coat a 24 -well plate with $0.1 \%$ gelatin $(250 \mu \mathrm{L} /$ well $)$ and incubate at $37{ }^{\circ} \mathrm{C}$.

2. Aspirate the fibroblast medium on the aHDFs. Wash once with DPBS. Dissociate the cells with $0.05 \%$ trypsin $\left(1.5 \mathrm{~mL}\right.$ per T75 flask) at $37^{\circ} \mathrm{C}$ for 3-5 min

3. Add fibroblast medium to neutralize the trypsin ( $3 \mathrm{~mL}$ per flush per $T 75$ flask) and collect the detached cells in a $15 \mathrm{~mL}$ tube by flushing out the cells in the flask twice.

4. Spin down the cells at $400 \times \mathrm{g}$ for $5 \mathrm{~min}$. Discard supernatant, and resuspend the cell pellet in $1 \mathrm{~mL}$ of fibroblast medium

5. Count the cells using an automated cell counter (to ensure a good quality conversion check that the cell viability is above $90 \%$ with trypan blue staining).

6. For a complete 24-well plate, prepare a suspension of $1,320,000$ cells in $13.2 \mathrm{~mL}$ of fibroblast medium to achieve a suspension of 100,000 cells $/ \mathrm{mL}$ of medium (or 55,000 cells/well in $550 \mu \mathrm{L}$ of fibroblast medium multiplied by the number of wells needed).

7. Aspirate the gelatin from the plate and wash twice with DPBS. Add $500 \mu \mathrm{L}$ of the cell suspension to each well and incubate overnight at $37{ }^{\circ} \mathrm{C}$ in $5 \% \mathrm{CO}_{2}$.

\section{Viral Transduction (Day 0)}

NOTE: Working with lentiviral particles requires category 2 equipment and the use of an agent to neutralize the virus. Wearing double pairs of gloves is also strongly recommended.

1. Warm up $13.2 \mathrm{~mL}$ of fibroblast medium to $37^{\circ} \mathrm{C}$.

2. Thaw a lentiviral vector containing the transcription factors Ascl1 and Brn2 with two short hairpin RNAs (shRNA) targeting REST at room temperature.

NOTE: Refer to reference ${ }^{8}$; the construct is available in a plasmid repository. For details on the procedure to produce lentiviruses please refer to reference ${ }^{9}$

3. Add the necessary volume of lentivirus to infect the aHDFs at multiplicity of infection (MOI) of 20 to the medium without any transduction enhancers.

Volume of virus $(\mu \mathrm{L})=\frac{\text { Number of cells to infect } \times \text { MOI } \times 1,000 \mu \mathrm{L}}{\text { Virus titer }(\mathrm{pfu})}$

4. Replace the medium in the 24 -well plate with fibroblast medium containing the lentiviral vector $(500 \mu \mathrm{L} /$ well $)$ and incubate overnight at $37^{\circ} \mathrm{C}$ in $5 \% \mathrm{CO}_{2}$.

5. The next day, replace the medium in the wells with fresh fibroblast medium without the lentiviral vector. 
NOTE: The medium is considered infectious for 7 days and as such, adequate protection and handling procedures should be used during the first week following viral transduction.

\section{Maintenance of the Converting Cells}

NOTE: Once conversion begins cells are susceptible to lifting; take care to tip the plate up and use a 1,000 $\mu \mathrm{L}$ pipette when removing media to avoid cells detaching.

1. On day 3 , remove the fibroblast medium and add $500 \mu \mathrm{L}$ of early neuronal conversion medium (see Table 1).

2. Two to three times a week, take out $225 \mu \mathrm{L}$ of old medium from the well and add-in $250 \mu \mathrm{L}$ of fresh early neuronal conversion medium.

3. On day 18 , remove all of the medium from each well and replace with $500 \mu \mathrm{L}$ of late neuronal conversion medium (see Table 1).

4. Continue to change half of the medium as above with late neuronal conversion medium every $2-3$ days until day 25 or experiment endpoint (Figure 1A).

NOTE: If cells are not plated in every well for the experiment, fill empty wells with PBS or water to prevent overt evaporation of the medium and minimize variation between wells.

\begin{tabular}{|c|c|c|}
\hline & Stock Concentration & Working Concentration \\
\hline \multicolumn{3}{|l|}{ Fibroblast medium } \\
\hline Basal medium & $\mathrm{N} / \mathrm{A}$ & $N / A$ \\
\hline Penicillin/Streptomycin & $10,000 \mathrm{U} / \mathrm{mL}$ & $100 \mathrm{mg} / \mathrm{mL}$ \\
\hline FBS & $\mathrm{N} / \mathrm{A}$ & $10 \%$ \\
\hline \multicolumn{3}{|l|}{ Freezing medium } \\
\hline Fibroblast medium & $\mathrm{N} / \mathrm{A}$ & $45 \%$ \\
\hline FBS & $\mathrm{N} / \mathrm{A}$ & $45 \%$ \\
\hline DMSO & $\mathrm{N} / \mathrm{A}$ & $10 \%$ \\
\hline \multicolumn{3}{|l|}{ Early neuronal conversion medium (ENM) } \\
\hline Neural differentiation medium & $\mathrm{N} / \mathrm{A}$ & N/A \\
\hline Penicillin/Streptomycin & $10,000 \mathrm{U} / \mathrm{mL}$ & $100 \mathrm{mg} / \mathrm{mL}$ \\
\hline CHIR99021 & $10 \mathrm{mM}$ & $2 \mu \mathrm{M}$ \\
\hline SB-431542 & $20 \mathrm{mM}$ & $10 \mu \mathrm{M}$ \\
\hline Noggin & $100 \mu \mathrm{g} / \mathrm{mL}$ & $0.5 \mu \mathrm{g} / \mathrm{mL}$ \\
\hline LDN-1931189 & $10 \mathrm{mM}$ & $0.5 \mu \mathrm{M}$ \\
\hline VPA & $1 \mathrm{M}$ & $1 \mathrm{mM}$ \\
\hline LM-22A4 & $20 \mathrm{mM}$ & $2 \mu \mathrm{M}$ \\
\hline GDNF & $20 \mu \mathrm{g} / \mathrm{mL}$ & $2 \mathrm{ng} / \mathrm{mL}$ \\
\hline NT3 & $10 \mu \mathrm{g} / \mathrm{mL}$ & $10 \mathrm{ng} / \mu \mathrm{L}$ \\
\hline db-cAMP & $50 \mathrm{mM}$ & $0.5 \mathrm{mM}$ \\
\hline \multicolumn{3}{|l|}{ Late neuronal conversion medium (LNM) } \\
\hline Neural differentiation medium & $\mathrm{N} / \mathrm{A}$ & N/A \\
\hline Penicillin/Streptomycin & $10,000 \mathrm{U} / \mathrm{mL}$ & $100 \mathrm{mg} / \mathrm{mL}$ \\
\hline LM-22A4 & $20 \mathrm{mM}$ & $2 \mu \mathrm{M}$ \\
\hline GDNF & $20 \mu \mathrm{g} / \mathrm{mL}$ & $2 \mathrm{ng} / \mathrm{mL}$ \\
\hline NT3 & $10 \mu \mathrm{g} / \mathrm{mL}$ & $10 \mathrm{ng} / \mu \mathrm{L}$ \\
\hline db-cAMP & $50 \mathrm{mM}$ & $0.5 \mathrm{mM}$ \\
\hline \multicolumn{3}{|l|}{ FACS Buffer } \\
\hline HBSS 1x [-calcium, -magnesium, - phenol red] & $\mathrm{N} / \mathrm{A}$ & N/A \\
\hline BSA & $\mathrm{N} / \mathrm{A}$ & $1 \%$ \\
\hline DNAase & $\mathrm{N} / \mathrm{A}$ & $0.05 \%$ \\
\hline
\end{tabular}

Table 1: Composition of the different media used. Full description of the composition for all media needed in this protocol including fibroblast medium, freezing medium, early neuronal conversion medium, late neuronal conversion medium, and FACS buffer. 


\section{Glass Coverslips for Electrophysiological Recordings}

NOTE: It is recommended to wear a lab coat, goggles, double gloves, and complete all of the work in a fume hood. This protocol is adapted from 10

1. Place glass coverslips at the bottom of a glass dish without overlap.

2. Add general lab cleaning detergent to submerge all of the coverslips without risking overflow during shaking (approximately $30 \mathrm{~mL}$ ). Place onto an orbital shaker at slow speed for $2 \mathrm{~h}$.

3. Wash six times (30 min each) with autoclaved deionized water.

4. Add $95 \%$ ethanol for $2 \mathrm{~h}$.

5. Remove the ethanol and wait until coverslips are dry.

6. Once dry, transfer the coverslips into a glass beaker and add $70 \%$ nitric acid until the coverslips are submerged.

7. Place the glass beaker into a sonicator bath for $60 \mathrm{~min}$.

8. Remove the nitric acid and wash three times with autoclaved deionized water. Caution: Always add acid to water, never the other way around as this can lead to a violent reaction.

9. Remove as much water from the beaker as possible and add concentrated hydrochloric acid $(\mathrm{HCl})$ until the coverslips are submerged; swirl the beaker and cover with paraffin film.

10. Sonicate for $60 \mathrm{~min}(50-60 \mathrm{~Hz}, 30 \mathrm{~W})$.

11. Remove as much $\mathrm{HCl}$ from coverslips as possible and place into an appropriate waste bin. Rinse with autoclaved deionized water twice.

12. Take the coverslips out of the hood and rinse 20 times (or more) with autoclaved deionized water until all of the $\mathrm{HCl}$ is removed.

13. Once dry, place coverslips into sterile 24 -well plates.

14. Place the plates under ultraviolet (UV) light overnight. The next day the coverslips are ready to use. NOTE: If glass coverslips are being used, it is recommended to use the PFL coating. Simply place one coverslip into each well of a 24-well plate (using sterile forceps) and follow the protocol as below.

\section{PFL Coating for Long Term Culture}

1. Coat the 24 -well plate with poly-L-ornithine $(500 \mu \mathrm{L} /$ well $)$ and leave overnight at $37{ }^{\circ} \mathrm{C}$ in $5 \% \mathrm{CO}_{2}$.

2. Aspirate the poly-L-ornithine and wait until it is dry enough to form drops on top.

3. Make a drop (approximately $60 \mu \mathrm{L}$ ) of laminin in the center of each well and spread to cover the entire surface of the coverslip. Leave for $2 \mathrm{~h}$ 45 min at $37^{\circ} \mathrm{C}$ in $5 \% \mathrm{CO}_{2}$.

4. Wash three times with DPBS.

5. Add fibronectin $(500 \mu \mathrm{L} /$ well $)$ and leave overnight at $37^{\circ} \mathrm{C}$ in $5 \% \mathrm{CO}_{2}$.

6. Wash once with DPBS before adding cells. NOTE: The PFL coating can be used for long term cultures of iNs (over 100 days), although progressive cell death is to be expected from day 30.

\section{FACS}

NOTE: To re-plate the cells after FACS sorting prepare a PFL-coated plate $48 \mathrm{~h}$ in advance. Cells can be sorted using a neural cell adhesion molecule (NCAM) antibody from day 20 onwards following transduction.

1. Remove the media with a $1,000 \mu \mathrm{L}$ pipette (do not wash to avoid cells detaching).

2. Add cell dissociating agent $(250 \mu \mathrm{L} /$ well $)$, leave for $10-20 \mathrm{~min}$ until the cells lift, and float as single cells

3. During this time prepare the FACS buffer.

4. Triturate gently with a $1,000 \mu \mathrm{L}$ pipette. If some clumps remain, incubate a little bit longer

5. Once a single cell suspension is obtained, remove it from the well and place into a $1.5 \mathrm{~mL}$ tube.

6. Flush out the well twice with late neuronal conversion medium and place into the same $1.5 \mathrm{~mL}$ tube.

7. Spin down at $400 \times \mathrm{g}$ for $5 \mathrm{~min}$ and discard the supernatant.

8. Resuspend the cell pellet in $200 \mu \mathrm{L}$ of FACS buffer.

9. Spin down the cells at $400 \times \mathrm{g}$ for $5 \mathrm{~min}$ and discard the supernatant.

10. Repeat steps 8.8 and 8.9 twice.

11. Resuspend the cell pellet in $50 \mu \mathrm{L}$ of FACS buffer containing human CD56 (NCAM) antibody at a concentration of 1:50 for 15 min on ice. Protect from light.

12. Spin down the cells at $400 \times \mathrm{g}$ for $5 \mathrm{~min}$

13. Resuspend in $200 \mu \mathrm{L}$ FACS buffer to wash and spin down the cells at $400 \mathrm{xg}$ for $5 \mathrm{~min}$.

14. Repeat step 8.13.

15. Resuspend in $200 \mu \mathrm{L}$ of FACS buffer containing propidium iodide (PI; $10 \mu \mathrm{g} / \mathrm{mL}$ ). Sort the NCAM positive (iNs) and PI negative (live) cells using FACS by gating based on the fluorescence intensity level of control samples that were not stained with the NCAM antibody or PI.

16. Collect the NCAM positive cells in a tube containing late neuronal conversion medium.

17. Count the cells using an automated cell counter and re-plate the cells at a high density $\left(50,000 \mathrm{cells} / \mathrm{cm}^{2}\right)$ in late neuronal conversion medium in a PFL coated dish.

18. Continue to change half of the medium 2-3 times a week with late neuronal conversion medium until the experiment endpoint (Figure 1A). 


\section{Representative Results}

A clear change in cell morphology should be visible from day 5 onwards (Figure 1B). Some cell death is to be expected after viral transduction, although not overtly. From each well in a 24-well plate a total cell yield of 20,000-40,000 cells should be expected by day 25 , of which about half should have become neurons. It is important to note that the yield and purity can vary across cell line, as well as with disease state and virus batches.

Cells will express most standard neuronal markers including MAP2 and TAU (Figure 1C) at day 25 in addition to exhibiting a mature neuronal morphology. It is possible to get a pure iN population by doing FACS based on the marker NCAM (see references ${ }^{8,11}$ ). Cells can thereafter be either re-plated onto PFL triple coating (see reference ${ }^{10}$ ) or directly frozen for biomolecular analyses.

If the cells are not sorted, immunofluorescence labeling with either MAP2 or TAU should be performed to identify the successfully converted cells and co-labeled with the protein of interest. 
A
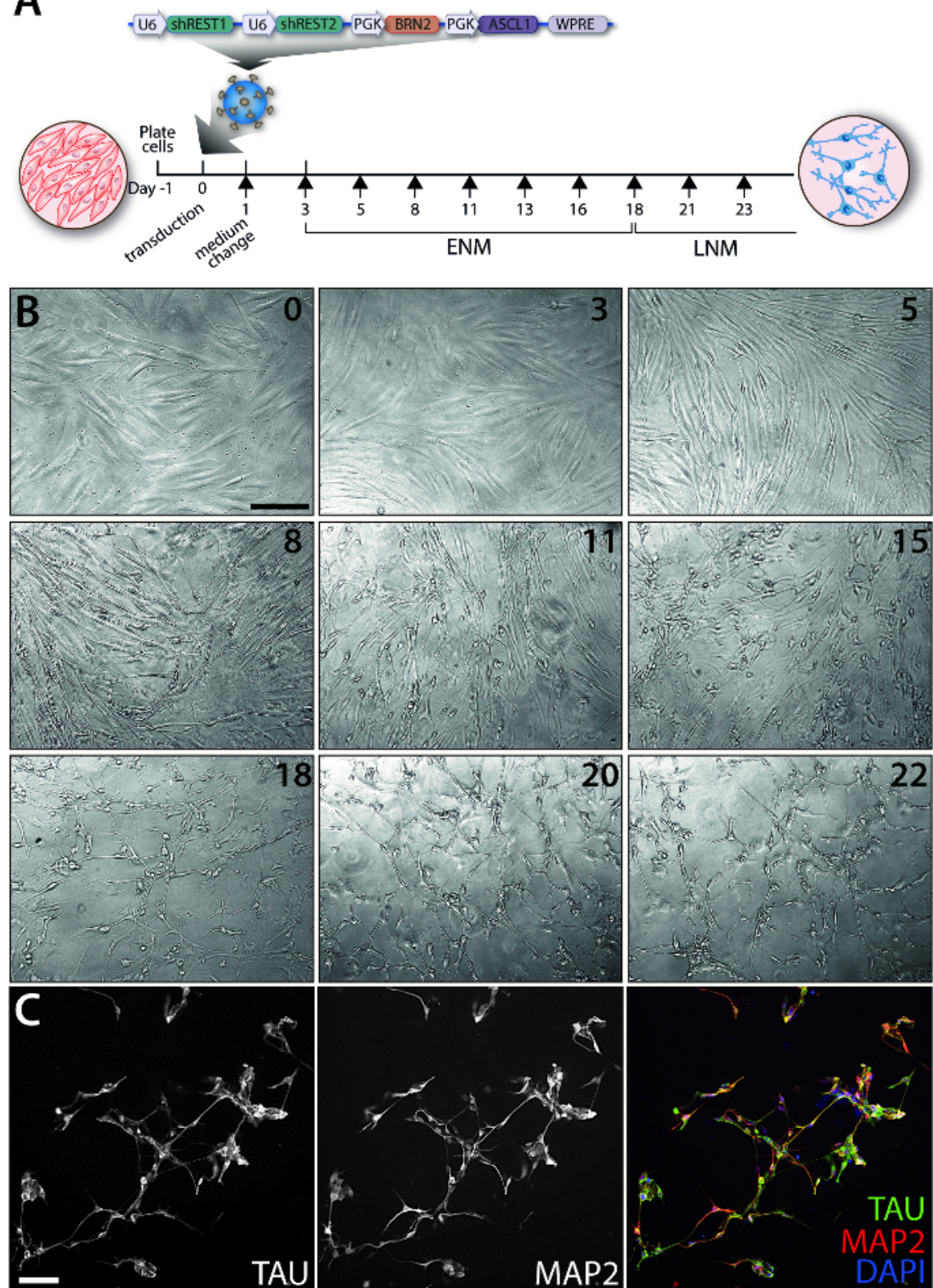

Figure 1: Evolution of the iN conversion over time. (A) Timeline of the experiment and map of the construct packaged in a lentivirus used to reprogram the adult human dermal fibroblasts. Each black arrow represents a medium change. (B) Representative phase contrast images depicting the changes in morphology of cells during the conversion process between day 0 to day 22 (as indicated on the upper right corner of each panel). Images were taken on a phase contrast microscope using the 10X objective. (C) Immunofluorescence image of a TAU and MAP2 double staining at day 35 post-transduction. Cells were fixed in $4 \%$ paraformaldehyde and permeabilized with $0.1 \%$ Triton in DPBS for 10 min. Cells were blocked for $30 \mathrm{~min}$ in a $5 \%$ serum solution in DPBS. The antibodies were diluted in blocking solution and applied overnight at $4{ }^{\circ} \mathrm{C}$. Fluorophore-conjugated secondary antibodies were diluted in blocking solution and applied for $2 \mathrm{~h}$. Cells were counterstained with DAPI for 15 min followed by 3 washes with DPBS. Images were taken on an inverted fluorescence microscope using the $20 \mathrm{X}$ objective. Scale bars $=100 \mu \mathrm{m}$ $(B, C)$. Abbreviations: ENM: early neuronal medium; LNM: late neuronal medium. Please click here to view a larger version of this figure.

\section{Discussion}

This one-step/one vector reprogramming method provides an efficient way to obtain iNs from human adult fibroblasts. Human adult fibroblasts are normally much more difficult to covert than fetal fibroblasts, with limited studies previously reporting efficiencies of approximately $5-10 \%{ }^{12,13}$ However, with this new protocol it is possible to achieve a neuronal yield (measured as MAP2+ cells) of approximately $50 \%{ }^{8}$. Additionally, our protocol can be used on dermal fibroblasts that have been passaged multiple times without losing efficiency of conversion. Thus far we have used cells passaged up to 14 times without detecting any decrease in conversion efficiency. Also, there is no difference in reprogramming efficiency in our hands with fibroblasts from donors of age between 52 and 87 . For more details on the age and disease of other cell lines tested with this construct see reference ${ }^{8}$. Other studies have also reported no difference in conversion efficiency using a lentiviral-based and small molecule-enhanced protocol with donors between 0 and 89 years ${ }^{4}$. Furthermore, consistent applicability with miRNA-based neuronal 
reprogramming has been reported in fibroblasts of all ages, with donors between 0 and 86 years ${ }^{7}$. Through this route, mature neurons can be obtained in approximately $12-15$ weeks in vitro or approximately 8 weeks following transplantation in vivo ${ }^{8}$. This is advantageous because it gives access to both disease and patient specific human iNs from tissue that is easily accessible. Although this protocol is efficient, it will not produce a $100 \%$ neuronal yield, and as such a purification step using FACS for instance is required.

The most critical step within this protocol is viral transduction (protocol section 4). It is crucial that the virus titer is precise, in addition to having plated an accurate number of aHDFs for conversion. The recommended titer for use with this protocol is between $4 \times 10^{8}$ and $4 \times 10^{9}$. Using a titer of anything below $1 \times 10^{8}$ would not be recommended as adding large volumes of virus will be toxic to the cells. Moreover, as the fibroblasts begin to covert to iNs they will become more fragile and susceptible to lifting. It is essential to be gentle when changing the media as not to disturb the cells too much. This can be done by removing the fluid slowly with a 1,000 $\mu \mathrm{L}$ pipette. Finally, when plating for reprogramming (protocol section 3 ) it is important to ensure a healthy fibroblast population before beginning an experiment; this is indicated by a cell viability of above $90 \%$ with trypan blue staining. The aHDFs should always be passaged before reaching $95 \%$ confluency.

If there is noticeable cell death before viral transduction, do not begin conversion: double check that the cell viability is above $90 \%$ and that there were no problems with coating of the plate. It is expected to have a small amount of cell death following viral transduction, however, this should not be significant. In this case, confirm accurate seeding of 50,000 aHDFs/well and check the virus titer. If there is noticeable inconsistency between wells during conversion, first check that every well contains an equal amount of media and overt evaporation is not occurring at the edges (if necessary extra media can be added to the edge wells). Alternatively, check step 4.4, and ensure appropriate mixing for a homogenous suspension when transducing. It is crucial to add the lentivirus to the medium first, before adding this into the wells. Directly adding lentivirus into the wells will increase well to well variability and is also likely to be toxic to the cells. Lastly, always check that the medium is warmed to $37^{\circ} \mathrm{C}$ before adding to cells.

This protocol includes optional sections for coating conditions for long term culture of iNs and FACS sorting to increase neuronal purity. For experiments wishing to investigate functional characterization of iNs, a protocol for preparation of glass coverslips for electrophysiology has also been included. The conversion protocol here is set up for use with a 24-well plate; if desired this can be modified to a 6-, 12-, 48-, 96-well plate or flasks. In this case, please adjust all volumes to the surface area of the plate or flask utilized.

This protocol uses the forced expression of Ascl1 and Brn2 in combination with a REST knockdown all packaged in one single vector ${ }^{8}$ to generate iNs of a pan-neuronal phenotype. The generation of any glial subtypes with this method, however, has not been assessed. This method would thus need to be modified for use with other reprogramming factors to obtain subtype specific neurons. Direct reprogramming has previously shown the possibility to generate motor neurons, sensory neurons, photoreceptors, striatal medium spiny neurons, and dopaminergic neurons $^{10,14}$. This will be beneficial when investigating neurological diseases in which specific neuronal subtype are preferentially affected, for example Parkinson's disease and dopaminergic neurons.

Until very recently, direct neural reprogramming technology did not allow for the production of iNs in a standardized and efficient manner to a level that is required for toxicology and drug screening assays on a large scale. This new method is very efficient and can be used on fibroblasts that have been passaged many times, such that it now removes these restrictions and opens up for a vast array of studies, not only in a human neural system, but also in a system that can be patient specific. The simplicity of this approach renders the iN technology accessible for any groups wanting to perform similar studies in-house and can be easily used not only for large scale biomedical applications, such as drug screening and toxicology assays, but also to support data derived from animal models and human post mortem tissue samples.

\section{Disclosures}

The authors have nothing to disclose.

\section{Acknowledgements}

We thank Marie Persson Vejgården for technical assistance. The research leading to these results has received funding from the New York Stem Cell Foundation, the European Research Council under the European Union's Seventh Framework Programme: FP/2007-2013 Neuro Stem Cell Repair (no. 602278) and ERC Grant Agreement no. 30971, the Swedish Research Council (grant agreement 521-2012-5624, 2016-00873 and 70862601/ Bagadilico), Swedish Parkinson Foundation (Parkinsonfonden), and the Strategic Research Area at Lund University Multipark (multidisciplinary research in Parkinson's disease). Janelle Drouin-Ouellet is supported by a Canadian Institutes of Health Research (CIHR) fellowship (\#358492), and Roger Barker is supported by an NIHR Biomedical Research Centre grant to the University of Cambridge/ Addenbrooke's Hospital. Malin Parmar is a New York Stem Cell Foundation Robertson Investigator. Shelby Shrigley is funded by the European Union Horizon 2020 Programme (H2020-MSCA-ITN-2015) under the Marie Skłodowska-Curie Innovative Training Network and Grant Agreement No. 676408.

\section{References}

1. Takahashi, K., \& Yamanaka, S. Induction of pluripotent stem cells from mouse embryonic and adult fibroblast cultures by defined factors. Cell. 126, 663-676 (2006).

2. Takahashi, K. et al. Induction of pluripotent stem cells from adult human fibroblasts by defined factors. Cell. 131, 861-872 (2007).

3. Avior, Y., Sagi, I., \& Benvenisty, N. Pluripotent stem cells in disease modelling and drug discovery. Nat Rev Mol Cell Biol. 17, 170-182 (2016).

4. Mertens, J. et al. Directly Reprogrammed Human Neurons Retain Aging-Associated Transcriptomic Signatures and Reveal Age-Related Nucleocytoplasmic Defects. Cell Stem Cell. 17, 705-718 (2015).

5. Hashizume, O. et al. Epigenetic regulation of the nuclear-coded GCAT and SHMT2 genes confers human age-associated mitochondrial respiration defects. Sci Rep. 5, 10434 (2015). 
6. Lapasset, L. et al. Rejuvenating senescent and centenarian human cells by reprogramming through the pluripotent state. Genes Dev. $\mathbf{2 5}$, 2248-2253 (2011).

7. Huh, C. J. et al. Maintenance of age in human neurons generated by microRNA-based neuronal conversion of fibroblasts. Elife. 5, (2016).

8. Drouin-Ouellet, J. et al. REST suppression mediates neural conversion of adult human fibroblasts via microRNA-dependent and -independent pathways. EMBO Mol Med. (2017).

9. Tiscornia, G., Singer, O., \& Verma, I. M. Production and purification of lentiviral vectors. Nat Protoc. 1, 241-245 (2006).

10. Richner, M., Victor, M. B., Liu, Y., Abernathy, D., \& Yoo, A. S. MicroRNA-based conversion of human fibroblasts into striatal medium spiny neurons. Nat Protoc. 10, 1543-1555 (2015).

11. Lau, S., Rylander Ottosson, D., Jakobsson, J., \& Parmar, M. Direct neural conversion from human fibroblasts using self-regulating and nonintegrating viral vectors. Cell Rep. 9, 1673-1680 (2014).

12. Pfisterer, U., Wood, J., Nihlberg, K., Hallgren, O., Bjermer, L., Westergren -Thorsson, G., Lindvall, O., \& Parmar, M. Efficient induction of functional neurons from adult human fibroblasts. Cell Cycle. 10, 3311-3316 (2011).

13. Caiazzo, M., Dell'Anno, M.T., Dvoretskova, E., Lazarevic, D., Taverna, S., Leo, D., Sotnikova, T.D., Menegon, A., Roncaglia, P., Colciago, G., Russo, G., Carninci, P., Pezzoli, G., Gainetdinov, R.R., Gustincich, S., Dityatev, A., \& Broccoli, V. Direct generation of functional dopaminergic neurons from mouse and human fibroblasts. Nature. 476, 224-227 (2011).

14. Masserdotti, G., Gascón, S., \& Götz, M. Direct neuronal reprogramming: learning from and for development. Development. 143, 2494-2510 (2016).

15. Pereira, M., Pfisterer, U., Rylander, D., Torper, O., Lau, S., Lundblad, M., Grealish, S., \& Parmar, M. Highly efficient generation of induced neurons from human fibroblasts that survive transplantation into the adult rat brain. Sci Rep. 4, 6330 (2014). 